\title{
EFFICIENCY ANALYSIS OF HOSPITALS IN PUNJAB DISTRICTS: AN APPLICATION OF DEA BOOTSTRAP
}

\author{
Nabila Asghar \\ Assistant Professor Department of Economics \& Business Administration, University of \\ the Education, Lahore, Pakistan \\ Hafeez-ur-Rehman \\ Professor of Economics, School of Business and Economics, University of Management and \\ Technology Lahore, Pakistan \\ Hafiz Ghulam Mujaddid \\ Associate Research Fellow at Punjab Economic Research Institute Lahore, Pakistan
}

\begin{abstract}
The main objective of this study is to analyze the efficiency of hospitals of districts of Punjab a province of Pakistan. The study uses output oriented DEA bootstrap technique formeasuring the efficiency of hospitals by utilizing four inputs and two outputs. The results of the study reveal that not a single district appears to be fully efficient when bias correctedefficiency technique is used. The overall efficiency which is measured in the form of mean efficiency have shown rising trend in 2011 and afterward little deterioration in 2013. The study identifies the less efficient and highly efficient districts of hospitals of Punjab province of Pakistan. The inference from this study may be helpful for policy makers to formulate and implement policies which help the hospitals of districts of Punjab to perform better throughimproving their efficiency.
\end{abstract}

Keywords: Technical efficiency, DEA bootstrap, Output, Hospitals

Jel Classification: C13; I11; L33

*The material presented by the author does not necessarily portray the view point of the editors and the management of the Ilma University - Formerly IBT

1. NabilaAsghar

Hafeez-ur-Rehman

3. Hafiz Ghulam Mujaddid
:Nabila.asghar@ue.edu.pk2.

: hafeez.rehman@umt.edu.pk

:hafizghulam@umt.edu.pk

CILMA-JBS is published by the Ilma University - Formerly IBT

Main Ibrahim Hydri Road, Korangi Creek, Karachi-75190, Pakistan 


\section{INTRODUCTION}

Health is an important factor in social sector of an economy which may have a substantial contribution in the overall economic well-being of any country (for details see Bloom et al, 2001, Akram et al, 2008). This indicates that for a country to achieve rapid economic development it is essential to introduce better health structure through providing improved, better and low cost health facilities to the people. Studies available in literature have pointed out that efficiency of health sector may be represented by the performance of hospitals. The efficiency analysis of hospitals plays a significant role in the assessment of health policies and comparative analysis of health sector (Biorn et al, 2003).

The main purpose of efficiency measurement is to determine whether entities are employing their resources in an efficient way or not.For measuring efficiency of an organization three criterion: profit maximization, cost minimization and output maximization have been utilized. According to Wholey and Hatry, (1992), the main purpose of measuring efficiency is to compare the different units, the earlier level of efficiency with the existing level of efficiency and the planned efficiency with the actual efficiency level. Furthermore, performance can be measured by comparing the efficiency of entities functioning under the similar conditions. An organization or entity is considered to be technically efficient if the maximum output has been produced from a given set of inputs or specific amount of output is produced from the minimum set of inputs.

The main objective of this study is to check whether health institutions in all the districts of Punjab deliver health services at an optimal level or not through measuring their efficiency and comparing it with other districts. For this purpose technical efficiency of hospitals of each district of Punjab is evaluated assuming each district as an entity or decision making unit (DMU). The present study uses output oriented variable returns to scale (VRS) for obtaining efficiency scores. The major feature of output oriented model is that different sets of output can be produced by utilizing the same set of inputs. This means maximum level of output can be obtained with fixed inputs through minimizing the inefficiencies. This study is highly important because there is hardly any study available in literature which hasestimated the technical efficiency of health institutions of every district of Punjab using DEA bootstrap technique. In output oriented DEA bootstrap technique efficiency score 1 indicates that the hospitals of specific district are fully efficient. The estimated efficiency score less than 1 means that the specific district is less efficient.The results of the study may help the responsible health administrators to pay proper attention to inefficient hospitals of districts of Punjaband other health institutions working in public sector. For analysis purpose the Punjab province is selected because it is one of the highest per capita income provinces of Pakistan. It has 2455 Basis Health Units (BHUs) and 293 Rural Health Centers (RHCs) in public sector.

The remaining study is organized as follows: Section II contains review of literature. Section III provides methodological framework and data. Empirical results of every district are presented in Section IV. Section V concludes.

\section{REVIEW OF LITERATURE}

Chang et al (2004) try to find out the relationship between hospital ownership and operating efficiency of Taiwan hospitals for the period of 1996 and 1997. For measuring the 
efficiency of hospitals non-parametric DEA approach has been used which is based on four inputs (no of beds, physicians, nurses and supporting medical personnel) and three outputs (no of patient days, outpatient and no of patient receiving surgery). The cross analysis has also been conducted between private and public regional and district hospitals. The results of the study reveal that private hospitals are more efficient than public hospitals in case of both regional and district hospitals of Taiwan.

Gannon (2005) measures the efficiency of Irish hospitals for the period of 1995 and 2000. Using DEA and SFA techniques to evaluate the variations in technical efficiency by utilizing the three inputs (no of beds, no of medical staff and no of non-medical staff) and three outputs (inpatients, outpatients and day cases). DEA technique provides efficiency scores 0.96 and 0.94 for the period of 1995 and 2000 respectively while, in case of SFA, the efficiency score are 0.63 and 0.60 for the period of 1995 and 2000 respectively. The study concludes that SFA has removed the random noise which was included in DEA.

Vitikainen et al, (2009) measure the efficiency of forty hospitals of Finlad for the period of 2005 using DEA technique. The study introduces two separate grouping system of output for comparing the activities and episode output estimates and between these two different output grouping system. The study concludes that efficiency estimates are highly sensitive to the grouping system and are not sensitive to the choices which are made between episode and activity description of output. Furthermore, large hospitals follow decreasing returns to scale. Tiemann and Schreyogg (2009) measure the efficiency of hospitals of Germany for the period 2002 and 2006. In first stage, the study uses DEA bootstrap approach for measuring the biased corrected technical efficiency utilizing the six inputs and two outputs for three types of hospitals. In the second stage, the study finds the determinants of the efficiency of hospital sector using the truncated regression. The results of the study reveal that public hospitals outperform significantly the private profit making and private non-profit making hospitals. The study concludes that hospital size has positive and significant impact on efficiency scores.

Moshiri et al. (2011) measure the technical efficiency of 24 clinical departments of three teaching hospitals in Malaysia for the period of 1998 to 2006. The study uses DEA technique to measure the efficiency utilizing four input variables (no of doctors, no of beds, no of nurses and no of non-medical staff) and two output variables (no of indoor patient and no of outdoor patient). The results of the study show that only three clinical departments are technically fully efficient out of 24 departments.

Varabyova and Schreyogg (2013) measure the technical efficiency of OECD countries and provide the cross country analysis of health sectors of these countries. In the first stage the study usesboth non-parametric (DEA) and parametric (SFA) techniques to measure the efficiency utilizing the four inputs (no of beds, total employment, physicians and nurses of hospitals) and two outputs (discharges and mortalities). In the second stage, several environmental variables were incorporated for analyzing their impact on efficiency. Spearman rank correlation test has been used to assess the internal and external significance of results of different models. The study concludes that increasing trend of health care expenditure per capita positively affects the efficiency of the health sectors in all OECD countries.

Gok and Sezen (2013) measure the efficiency of 348 hospitals of Turkey and also try to observe the impact of efficiency on patient satisfaction. The study uses DEA technique to 
measure the efficiency utilizing three inputs and seven outputs. The regression analysis has also been conducted to make sure the impact of efficiency, structural quality and institutional factors on patient satisfaction. The results of the study reveal that efficiency playsan important role in analyzing the association between structural quality and patient satisfaction. The study concludes that trade-off between quality and efficiency largely depends on the size of the hospital entity.

Rasool et al. (2014) measure the efficiency of the sixteen sub units of the private non-profit organization of Pakistan for the period of 2010. The study usesDEA approach to measure the efficiency utilizing the three inputs (no of Beds, Specialists and Nurses) and three outputs(Inpatient, Outpatient visits and Total number of surgery). The results of the study indicate that only 5 units appear to be fully efficient in case of constant return to scale(CRS) while 6 units turn up fully efficient in case of variable return to scale (VRS). The study concludes that mean efficiency of VRS is higher than the mean efficiency of CRS.

Bwana (2015) measures the efficiency of fifteen volunteering agency hospitals (VAHs) of Tanzania for the period of 2009 to 2012. The study employsthe DEA technique to measure the efficiency utilizing the three inputs (total surgeries, no of beds and full time staff) and two outputs (indoor patients and outdoor patients). The results of the study reveal that only four hospitals are technically efficient for the whole period of time. Furthermore, annually VAHs are $59.79 \%, 60.01 \%, 57.49 \%$ and $55.08 \%$ technically efficient for the period of 2009, 2010, 2011 and 2012 respectively. The study concludes that there is no increase in efficiency over the whole period of analysis.

Matranga and Spienza (2015) try to measure efficiency and the effect of institutional inappropriateness on efficiency evaluation in 116 short-term, acute-care hospitals of Sicily for the period of 2009. The study usesoutput oriented DEA technique and utilizes the four inputs (indoor patient beds, medical staff, nursing staff and other personnel) and four outputs (two desirable outputs, ordinary discharges and hospital admissions and two undesirable outputs, inappropriate discharges and day-hospitals). The study finds that Sicilian hospitals are $20 \%$ inefficient for the specific period of time.

The above mentioned studies reveal that there is only one study on the efficiency analysis of hospitals of Pakistan which covers just sub-centers of trusts and uses DEA technique for measuring efficiency. According to Simar and Wilson $(1998,2000)$ simple DEA is not appropriate technique due to various limitations such as efficiency scores are serially correlated when it is calculated by DEA and this approach does not provide any interpretation of data generating process (DGP). Furthermore, it has uncertainty about what is being estimated and also conventional inference methods which are used in the DEA are inconsistent and invalid. This study uses DEA bootstrap approach and it measures the bias corrected technical efficiency for hospitals of 35 districts of Punjab by considering each district as one decision making unit (DMU) and also this techniqueproduces the confidence interval. The study will also provide efficiency estimates of DEA approach for comparing the results of both techniques for interested readers.

\section{METHODOLOGICAL FRAMEWORK AND DATA COLLECTION}

The concept of efficiency measurement was introduced by Farrel (1957). For measuring efficiency two relevant approaches exist in the literature: Parametric and Non- 
parametric. Parametric approach can be used to specify and estimate a parametric production function or cost frontier. The main feature of parametric (Stochastic Frontier Analysis) is that it allows incorporating its stochastic error for hypothesis testing. The major drawback of this approach is that it requires formulating explicit functional form along with the distributional assumption of error term. This indicates that Stochastic Frontier Analysis (SFA) is sensitive to the selection of the parametric functional form. While on the other hand, non-parametric approach also known as Data Envelopment Analysis (DEA) has advantage of not imposing prior parametric restrictions on the technology. Therefore, it is less sensitive to misspecification. Furthermore, it is not subject to distributional assumption of the error term. However, DEA being a deterministic approach assumes that all deviations from the estimated frontier are the results of inefficiency which make it sensitive to measurement errors and data noises.

Simar and Wilson $(1998,2000)$ point out that due to several restrictions DEA model fails to incorporate the data generating process (DGP). As a result the efficiency estimates obtained through DEA are serially correlated. This indicates that the general DEA estimates are statistically invalid. They also point out that DEA efficiency estimates are exaggerated and under estimate the frontier. They propose an alternative estimation technique called DEA bootstrap approach which is considered to be a better and significant approach for finding the bias corrected technical efficiency (T.E) scores. This method produces confidence intervals for the efficiency estimates which allow hypothesis testing.

\subsection{Choice of Inputs and Outputs}

Magnussen (1996) is of opinion that hospitals' efficiency results are sensitive to the selection of output. He points out that patients' days should not be considered as output for measuring efficiency of hospitals because it may give exaggerated results. He suggests that for avoiding this situation it is preferable to use number of patients' visit to hospitals as output.Keeping in view the above mentioned discussion, this study uses indoor and outdoor patients as output and number of doctors, number of beds, number of nurses and number of hospitals as inputs. The inclusion of number of hospitals in the list of inputs is likely to bring variations in the efficiency of every district. For measuring efficiency of hospitals of every district of Punjab the data on inputs and outputs have been collected from health department of government of Punjab for the period 2008, 2011 and 2013.

\section{EMPIRICAL ANALYSIS}

The results of VRS technical efficiency are presented in the Table 1 for the period of 2008,2011 and 2013 after 2500 bootstrapped iterations. District names are given in the firstcolumn, results of original DEA efficiency scores represented by DEA (Biased Efficiency)are shown in second column, biased corrected efficiency scores represented by the biased corrected (DEA Bootstrap) are specified in third column while lower bound (LB) and upperbound (UB) of confidence interval are presented in fourth and fifth column respectively forthe period of 2008, 20011 and 2013.

The estimates presented in Table 1 show that the original efficiency scores (DEA) over estimate the results and underestimate the frontier. DEA scores do not fall in the confidence interval and these efficiency estimates are beyond the confidence interval due to the presence of bias. These results are consistent with the limitations of DEA described by Simar and Wilson (2000). Biased corrected technical efficiencies (DEA bootstrap) have been estimated 
after 2500 iterations, which eliminates the bias of exaggeration from the results. This estimation technique provides better and appropriate results as compare to DEA technique because these results fall in the confidence interval.

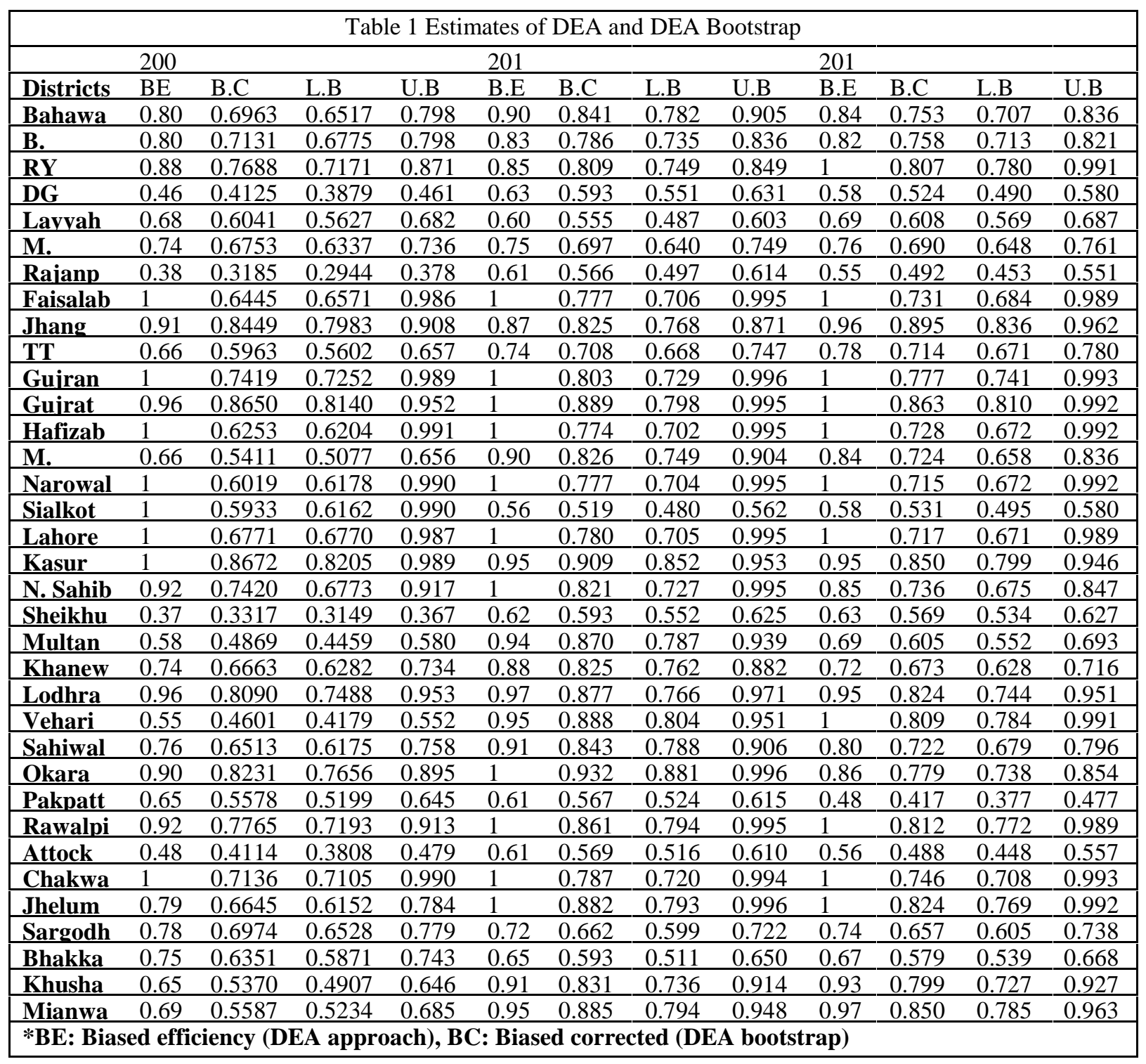

The efficiency scores of DEA technique for the period 2008 reveal that eight hospital disctricts are fully efficient. Bias corrected efficiency scores (DEA bootstrap) show that there is not even a single hospitals' district which is fully efficient. The estimates of this technique show that in 2008 Kasur district is almost 86\% efficient and with the help of same inputs $14 \%$ efficiency can be enhanced. Furthermore, Rajanpur district has shown the lowest efficiency which is almost $38 \%$ only. This indicates there is a huge room for increasing the efficiency of Ranjanpur district with the same inputs.

According to DEA technique eleven hospitals of districts of Punjab are fully efficient in 2011. While on the other hand the results of bias corrected efficiency score (DEA bootstrap) show that again there is not even a single district of hospitals fully efficient in 2011. District Okara appears to be highly efficient with efficiency score of 0.93 . Furthermore, out of 35 districts 22 districts gain technical efficiency and 13 districts lose minor technical efficiency. In 2013, DEA technique reveals that 11 districts of hospitals are fully efficient. According to 
corrected efficiency score (DEA bootstrap) not a single hospitals district is fully efficient. Jhang appears to be highly efficient district with score of 0.89 . This indicates that in this district there is a potential of increasing efficiency by $11 \%$ through using the existing levels of inputs.

The overall efficiency levels of all hospitals of districts can be observed through their mean values. The estimates of mean value of both DEA and DEA (bootstrap) for the period 2008, 2011 and 2013 are presented in Table 2 . These results provide interesting and highly valuable information regarding the existing efficiency and potential of increasing efficiency using the exiting inputs. The results of both DEA and DEA (bootstrap) approaches show that overall mean efficiencies of hospitals of districts rise from 2008 to 2011 and then decline in 2013. The DEA approach shows that the estimates of overall efficiency level of all hospitals of districts of Punjab are 0.786, 0.858 and 0.837 for the period 2008, 2011 and 2013 respectively. The DEA bootstrap approach shows that the overall efficiency level of all hospitals of districts of Punjab is 0.637 for the period of 2008 which means that overall hospitals of districts of Punjab are $63.7 \%$ efficient and almost $36 \%$ efficiency can be improved with the existing level of inputs. In year 2011, the overall efficiency of all Punjab districts has shown an improvement as compare to 2008. The overall efficiency score appears to be 0.763 which means that all the districts are $76.3 \%$ efficient and still there is a capacity of improvement of almost $23 \%$. In year 2013, there is a deterioration of the overall efficiency levels to 0.708 . This indicates that there exists potential of almost $29 \%$ increase in overall efficiencies with the existing levels of inputs.

Table 2 Mean efficiencies of hospitals' districts

\begin{tabular}{|lll|}
\hline & DEA & B.C \\
\hline $\mathbf{2 0 0 8}$ & 0.786683 & 0.637466 \\
\hline $\mathbf{2 0 1 1}$ & 0.85828 & 0.763904 \\
\hline $\mathbf{2 0 1 3}$ & 0.837343 & 0.708125 \\
\hline
\end{tabular}

\section{CONCLUSION}

Health being an indispensable factor in human life plays a significant role in achieving the goal of overall economic well-being in an economy. The existence of better health structure is considered to be a necessary condition for achieving rapid pace of economic development through utilizing the existing resources properly. The performance of hospitals can be observed through the efficiency of health sector and this study is an attempt to observe the performance of hospitals of districts of Punjab.

The present study tries to measure the efficiency of hospitals of districts of Punjab through measuring the technical efficiency of 35 districts of Punjab. For this purpose bias corrected efficiency technique (DEA bootstrap) has been utilized which is considered to be more appropriate than other techniques available in the literature. The present study shows the cross analysis of the districts within one period of time and district to district in three time periods.

The estimates of bias corrected efficiencies (DEA bootstrap) show that on the whole period of estimation none of the hospitals of districts is technically fully efficient. Furthermore, the 
overall efficiency measured by both DEA and DEA bootstrap techniques have shown increasing trend in 2011 but decline in efficiency is observed in 2013. From the above discussion it can be concluded that still there is a room to improve the overall efficiency of the hospitals of districts of Punjab with the existing levels of inputs. The present study points out that three hospitals of districts of Punjab Kasur, Okara and Jhang are highly efficient for the period 2008, 2011 and 2013 respectively. The identification of less efficient hospitals of districts of Punjab helps the policy makers and health administration to pay proper attention to these hospitals of districts of Punjab. For this purpose there is a need to formulate and implement appropriate policies for increasing the efficiency of less efficient hospitals of districts of Punjab.

\section{REFERENCES}

Akram, N., IhtshamulHaqPadda, \& Khan, M. (2008). The long term impact of health on economic growth in Pakistan. The Pakistan Development Review, 47(4), 487-500.

Biorn, E., Hagen, T. P., Iversen, T., \& Magnussen, J. (2003). The effect of activity-based financing on hospital efficiency: a panel data analysis of DEA efficiency scores 19922000. Health Care Management Science, 6(4), 271-283.

Bloom, D. E., Canning, D., \&Sevilla, J. (2001). The effect of health on economic growth: theory and evidence (No. w8587). National Bureau of Economic Research.

Bwana, K. M. (2015). Measuring Technical Efficiency of Faith Based Hospitals in Tanzania: An application of Data Envelopment Analysis (DEA). Research in Applied Economics, 7(1), 1-12.

Chang, H., Cheng, M. A., \& Das, S. (2004). Hospital ownership and operating efficiency: evidence from Taiwan. European Journal of Operational Research, 159(2), 513-527.

Farrell, M. J. (1957). The measurement of productive efficiency. Journal of the Royal Statistical Society. Series A (General), 120(3), 253-290.

Gannon, B. (2005). Testing for variation in technical efficiency of hospitals in Ireland. Economic and Social Review, 36(3), 273.

Gok, M. S., \&Sezen, B. (2013). Analyzing the ambiguous relationship between efficiency, quality and patient satisfaction in healthcare services: The case of public hospitals in Turkey. Health policy, 111(3), 290-300.

Magnussen, J. (1996). Efficiency measurement and the operationalization of hospital production. Health services research, 31(1), 21-37.

Matranga, D., \& Sapienza, F. (2015). Congestion analysis to evaluate the efficiency and appropriateness of hospitals in Sicily. Health Policy, 119(3), 324-332.

Moshiri, H., Aljunid, S. M., Amin, R. M., Dahlui, M., \& Ibrahim, W. N. (2011). Measuring efficiency of teaching hospitals in Malaysia. International Journal of Business and Management, 6(4), 207-2013.

Rasool, S. A., Saboor, A., \&Raashid, M. (2014). Measuring Efficiency of Hospitals by DEA: An Empirical Evidence from Pakistan. International Journal of Public Health Science (IJPHS), 3(2), 129-136.

Simar, L. and Wilson, P. (2000). Statistical Inference in Nonparametric Frontier Models: The State of the Art. Journal of Productivity Analysis, 13(1), 49-78.

Simar, L., \& Wilson, P. W. (1998). Sensitivity analysis of efficiency scores: How to bootstrap in nonparametric frontier models. Management science, 44(1), 49-61.

Tiemann, O., \&Schreyogg, J. (2009). Effects of ownership on hospital efficiency in Germany. BUR-Business Research, 2(2), 115-145. 
Varabyova, Y., \&Schreyogg, J. (2013). International comparisons of the technical efficiency of the hospital sector: Panel data analysis of OECD countries using parametric and non-parametric approaches. Health policy,112(1), 70-79.

Vitikainen, K., Street, A., \&Linna, M. (2009). Estimation of hospital efficiency-Do different definitions and casemix measures for hospital output affect the results? Health Policy, 89(2), 149-159.

Wholey, J. S., \&Hatry, H. P. (1992). The case for performance monitoring. Public Administration Review, 52(6) 604-610. 